\title{
A Characterization of Max-Min SIR-Balanced Power Allocation with Applications
}

\author{
Sławomir Stańczak ${ }^{\dagger}$, Michał Kaliszan ${ }^{\dagger}$, Nicholas Bambos* and Marcin \\ Wiczanowski ${ }^{\dagger}$ \\ ${ }^{\dagger}$ Fraunhofer German-Sino Lab for Mobile Communications \\ Einsteinufer 37, D-10587 Berlin, Germany \\ Email: \{stanczak, michal.kaliszan, marcin.wiczanowski\}@ @hi.fraunhofer.de \\ *Department of Electrical Engineering \\ 350 Serra Mall, Stanford University, Stanford, CA 94305 \\ Email: bambos@stanford.edu
}

\begin{abstract}
We consider a power-controlled wireless network with an established network topology in which the communication links (transmitter-receiver pairs) are corrupted by the co-channel interference and background noise. We have fairly general power constraints since the vector of transmit powers is confined to belong to an arbitrary convex polytope. The interference is completely determined by a socalled gain matrix. Assuming irreducibility of this gain matrix, we provide an elegant characterization of the max-min SIR-balanced power allocation under such general power constraints. This characterization gives rise to two types of algorithms for computing the max-min SIR-balanced power allocation. One of the algorithms is a utility-based power control algorithm to maximize a weighted sum of the utilities of the link SIRs. Our results show how to choose the weight vector and utility function so that the utility-based solution is equal to the solution of the max-min SIR-balancing problem. The algorithm is not amenable to distributed implementation as the weights are global variables. In order to mitigate the problem of computing the weight vector in distributed wireless networks, we point out a saddle point characterization of the Perron root of some extended gain matrices and discuss how this characterization can be used in the design of algorithms in which each link iteratively updates its weight vector in parallel to the power control recursion. Finally, the paper provides a basis for the development of distributed
\end{abstract}


power control and beamforming algorithms to find a global solution of the max-min SIR-balancing problem.

\section{Index Terms}

Max-min SIR-balancing, Max-min fairness, Power control, Wireless networks, Utility maximization, Interference management, Distributed algorithms

\section{INTRODUCTION}

Wireless channel is error-prone and highly unreliable being subject to several impairment factors that are of transient nature, such as those caused by co-channel interference or multipaths. Excessive interference can significantly deteriorate the network performance and waste scarce communication resources. For this reason, strategies for resource allocation and interference management are usually necessary in wireless networks to provide acceptable QoS levels to the users.

There are different mechanisms for resource allocation and interference management. Power control may play a central role in distributed wireless mesh networks, where, due to the lack of a central network controller, link scheduling strategies are notoriously difficult to implement. Thus, a reasonable approach is to avoid only strong interference from neighboring links, and then use an appropriate power control policy to manage the remaining interference in a network. In this paper, we focus on the power control problem, which addresses the issue of coordinating transmit powers of links such that the worst signal-to-interference ratio (SIR) balanced against some SIR targets attains its maximum. This so-called max-min SIR-balancing problem is a widely studied resource allocation problem for wireless networks (see, for instance, [1], [2], [3], [4], [5], [6], [7], [8], [9], [10], [11], [12] as well as [13, Sections 3.1 and 5.6], [14], [15, Section 5.6]). A key feature of this strategy is that any given SIR (signal-to-interference ratio) targets are feasible if and only if they are satisfied under a max-min SIR-balanced power allocation. Moreover, the notion of max-min SIR-balancing is closely related to max-min fairness, the most common notion of fairness. Note that since we focus on static wireless networks, transmit powers are to be periodically adjusted to changing channel and network conditions (dynamic power control). This in turn presumes a relatively low up to moderate network dynamics. In contrast, in highly dynamic wireless networks, one should consider resource allocation schemes 
for stochastic wireless networks [16], [17], [18], [19], [20], [21], [22], [23].

In the noiseless case, which is widely considered in the literature [1], [2], [3], [5], [4], [24], [25], [26]. (an overview can be found in [11], [27]) and where power constraints play no role in the analysis, it is widely known [11], [13, Sections 3.1] that any positive eigenvector of the (irreducible) gain matrix scaled by a diagonal matrix of given SIR targets is a solution to the max-min SIR-balancing problem. In [12], the problem was solved for a "noisy" downlink channel constrained on total power. The sum constraint on transmit powers was captured by an additional equation so that the optimal solution is characterized in terms of some unique eigenvector of a certain irreducible gain matrix of higher dimension (see also [13, pp. 111-113]).

Assuming an irreducible gain matrix, Sections III IV extend these results to any convex polytope as the constraint set to model constraints on transmit powers of the links. In addition to the analysis in a higher dimension (as in [12]), we also obtain an elegant characterization of the max-min SIR-balanced power vector from an eigenvalue problem of the same dimension as the original problem. These results were inspired by [28], where the authors used different tools to characterize rate region in interference channel with constrained power (see also the acknowledgments after the conclusions at the end of this paper).

In Section $\mathrm{V}$, we use the results of Sections IIIIV to establish a connection between the max-min SIR-balancing power control problem and the utility-based power control problem. Such a connection is known in the noiseless case [15, Section 5.9] and constitutes the starting point for the analysis in [14]. More precisely, we show how to choose the weight vector and utility function so that solving the problem of maximizing a weighted sum of the utilities of the SIRs leads to the max-min SIR-balancing solution. This result was used in [29] to solve the max-min SIR-balancing problem over the joint space of admissible power vectors and receive beamformers. Thus, the results of this paper provide key tools to generalize the results of [14] to noisy channels under general power constraints and to a larger class of utility functions.

An advantage of the utility-based approach is that there exist distributed power control schemes to compute the max-min SIR power vector, provided that each link knows how to select its weight [30], [31]. The problem is however that a desired weight vector is determined by positive left and right eigenvectors of some nonnegative matrix, so that the links cannot choose their weights independently. Thus, as neither the eigenvectors nor the corresponding matrices are a priori known at any node, the presented approach for computing the max-min SIR power allocation is 
still not amenable to implementation in decentralized wireless networks.

A basic idea to overcome or at least to alleviate this problem is to let each link iteratively update its weight vector in parallel to the power control recursion. In Section V-B, we point out that a saddle point characterization of the Perron roots of some nonnegative matrices. This characterization provides a basis for efficient saddle point algorithms converging to a maxmin SIR-balanced power vector. Basically, the idea is redolent of primal-dual algorithms that employ some optimization methods to minimize the Lagrangian over primal variables and to simultaneously maximize it over dual variables.

Before starting with the analysis, we introduce definitions, notation and state the max-min SIR-balancing problem.

\section{DEFINITIONS AND PROBLEM STATEMENT}

We consider a wireless network with an established network topology, in which all links share a common wireless spectrum. Let $K \geq 2$ users (logical links) compete for access to the wireless links and let $\mathcal{K}=\{1, \ldots, K\}$ denote the set of all users. The transmit powers $p_{k}, k \in \mathcal{K}$, of the users are collected in the vector $\mathbf{p}=\left(p_{1}, \ldots, p_{K}\right) \geq 0$, which is referred to as power vector or power allocation. The transmit powers are subject to power constraints so that $\mathbf{p} \in \mathrm{P}$ where 1

$$
\mathrm{P}=\left\{\mathbf{p} \in \mathbb{R}_{+}^{K}: \mathbf{C p} \leq \hat{\mathbf{p}}, \mathbf{C} \in\{0,1\}^{N \times K}\right\} \subset \mathbb{R}^{K}
$$

for some given $\hat{\mathbf{p}}=\left(P_{1}, \ldots, P_{N}\right)>0$ and $\mathbf{C}$ with at least one 1 in each column so that $\mathrm{P}$ is a compact set. Throughout the paper, we use $\mathcal{N}=\{1, \ldots, N\}$ where $N$ is the number of power constraints. The main figure of merit is the SIR at the output of each receiver given by

(A.1) $\operatorname{SIR}_{k}(\mathbf{p})=p_{k} / I_{k}(\mathbf{p}), k \in \mathcal{K}$, where the interference function $I_{k}$ is $I_{k}(\mathbf{p})=(\mathbf{V} \mathbf{p}+\mathbf{z})_{k}=$ $\sum_{l=1}^{K} v_{k, l} p_{l}+z_{k}$.

$\mathbf{V}:=\left(v_{k, l}\right) \in \mathbb{R}_{+}^{K \times K}$ is the gain matrix, $v_{k, l}=V_{k, l} / V_{k, k}$ if $l \neq k$ and 0 if $l=k$ where $V_{k, l} \geq 0$ with $V_{k, k}>0$ is the attenuation of the power from transmitter $l$ to receiver $k$. The $k$ th entry of $\mathbf{z}:=\left(z_{1}, \ldots, z_{K}\right)$ is $z_{k}=\sigma_{k}^{2} / V_{k, k}$, where $\sigma_{k}^{2}>0$ is the noise variance at the receiver output.

Let $\gamma_{1}, \ldots, \gamma_{K}>0$ be the SIR targets and let $\boldsymbol{\Gamma}=\operatorname{diag}\left(\gamma_{1}, \ldots, \gamma_{K}\right)$. We say that the SIR targets are feasible if there exists a power vector $\mathbf{p} \in \mathrm{P}$ (called a valid power vector) such that $\operatorname{SIR}_{k}(\mathbf{p}) \geq \gamma_{k}>0$

\footnotetext{
${ }^{1} \mathbb{R}_{+}, \mathbb{R}_{++}$are nonnegative and positive reals, respectively.
} 
Definition 1: Given any $\Gamma, \overline{\mathbf{p}}$ is said to be a max-min SIR-balanced power vector if

$$
\overline{\mathbf{p}}:=\arg \max _{\mathbf{p} \in \mathrm{P}} \min _{k \in \mathcal{K}}\left(\operatorname{SIR}_{k}(\mathbf{p}) / \gamma_{k}\right) .
$$

It is important to notice that the maximum in (2) exists as $\min _{k \in \mathcal{K}}\left(\operatorname{SIR}_{k}(\mathbf{p}) / \gamma_{k}\right)$ is continuous on the compact set P. Thus, $\gamma_{k}$ are not necessarily met under optimal power control (2). For this reason, $\gamma_{k}$ can also be interpreted as a desired SIR value of link $k$. A trivial but important observation is that $\overline{\mathbf{p}}>0$, allowing us to focus on $\mathrm{P}_{+}=\mathrm{P} \cap \mathbb{R}_{++}^{K}$.

\section{Some Preliminary ObSERVATIONS}

In general, $\overline{\mathbf{p}}$ of Definition 1 is not unique. For general power constraints, the uniqueness is ensured if $\mathbf{V}$ is irreducible [32], [33] since then the links are mutually dependent through the interference. In order to see this, notice that the problem (2) is equivalent to finding the largest positive threshold $t$ such that $t \leq \operatorname{SIR}_{k}(\mathbf{p}) / \gamma_{k}$ for all $k \in \mathcal{K}$ and $\mathbf{p} \in \mathrm{P}$. The constraints can be equivalently written in a matrix form as $\Gamma \mathbf{z} \leq\left(\frac{1}{t} \mathbf{I}-\boldsymbol{\Gamma V}\right) \mathbf{p}$ and $\mathbf{p} \in \mathrm{P}$. So, as $\mathbf{p}$ must be a positive vector, [15. Theorem A.51] implies that the threshold $t$ must satisfy

$$
\rho(\mathbf{\Gamma V})<1 / t
$$

Now, one particular solution to the max-min SIR-balancing problem (2) is $\overline{\mathbf{p}}^{\prime}$ given by

$$
\overline{\mathbf{p}}^{\prime}=\left(1 / t^{\prime} \mathbf{I}-\boldsymbol{\Gamma} \mathbf{V}\right)^{-1} \boldsymbol{\Gamma} \mathbf{z}, \overline{\mathbf{p}}^{\prime} \in \mathrm{P}
$$

where

$$
t^{\prime}=\arg \max _{t \geq 0} t \quad \text { s.t. } \quad(1 / t \mathbf{I}-\mathbf{\Gamma V}) \mathbf{p}=\mathbf{\Gamma z}, \mathbf{p} \in \mathrm{P} .
$$

Note that $\overline{\mathbf{p}}^{\prime}$ is a max-min SIR-balanced power vector such that $\operatorname{SIR}_{k}\left(\overline{\mathbf{p}}^{\prime}\right) / \gamma_{k}=\operatorname{SIR}_{l}\left(\overline{\mathbf{p}}^{\prime}\right) / \gamma_{l}$ for each $k, l \in \mathcal{K}$. This immediately follows from (4) when it is written as a system of $K$ SIR equations. By (3), (4), (5) and [15, Theorem A.51], $\overline{\mathbf{p}}^{\prime}>0$ exists and is a unique power vector corresponding to a point in the feasible SIR region ${ }^{2}$ that is farthest from the origin in a direction of the unit vector $\gamma /\|\gamma\|_{1}$.

The above considerations are illustrated in Fig. 1] The plots depict two examples of feasible SIR regions in a system with two users. In both cases, the point $\left(\operatorname{SIR}\left(\overline{\mathbf{p}}^{\prime}\right)_{1}, \operatorname{SIR}\left(\overline{\mathbf{p}}^{\prime}\right)_{2}\right)$, with $\overline{\mathbf{p}}^{\prime}$

\footnotetext{
${ }^{2}$ The feasible SIR region is the subset of $\mathbb{R}_{+}^{K}$ of all SIR levels that can be achieved by means of power control. F defined by (7) becomes the feasible SIR region if $\phi(x)=x$ and $\gamma_{k}=1, k \in \mathcal{K}$.
} 

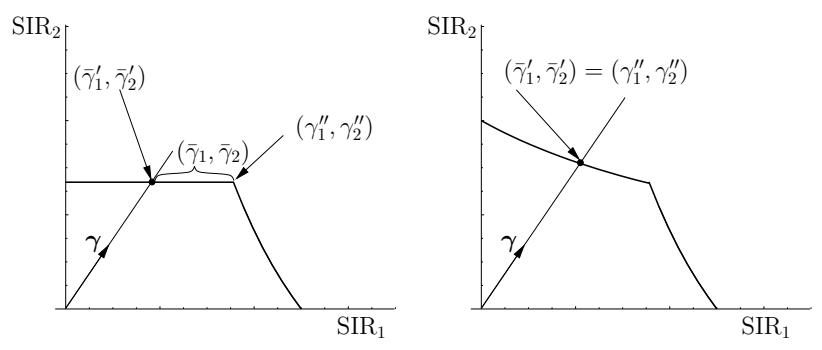

Fig. 1. The feasible SIR region under individual power constraints and two different gain matrices $\mathbf{V} \geq 0$. The following notation is used: $\bar{\gamma}_{k}=\operatorname{SIR}_{k}(\overline{\mathbf{p}})$ and $\bar{\gamma}_{k}^{\prime}=\operatorname{SIR}_{k}\left(\overline{\mathbf{p}}^{\prime}\right)$ where $\overline{\mathbf{p}}$ and $\overline{\mathbf{p}}^{\prime}$ are defined by (2) and 44, respectively. Left: $\mathbf{V}$ is chosen so that $\operatorname{SIR}_{2}(\mathbf{p})=p_{2} / z_{1}$, in which case $\overline{\mathbf{p}}$ is not unique. Right: $\mathbf{V}$ is irreducible, in which case $\overline{\mathbf{p}}$ is unique and equal to (4). The point $\left(\gamma_{1}^{\prime \prime}, \gamma_{2}^{\prime \prime}\right)$ corresponds to the max-min fair power allocation.

given by (4) is the point at the intersection of the boundary of the feasible SIR region with the line defined by the $\gamma$ vector and is max-min SIR-balanced. It is however not the unique solution if SIRs of some users can be increased without affecting the minimum. The examples of configurations in which the power allocation $\overline{\mathbf{p}}^{\prime}$ given by (4) is not and is a unique solution of the max-min SIR-balancing problem are presented in the left and in the right subplot of Fig. 1. respectively.

Now let us assume throughout the paper that (A.2) $\phi: \mathbb{R}_{++} \rightarrow \mathrm{Q} \subseteq \mathbb{R}$ is continuously differentiable and strictly increasing function. By strict monotonicity, one has $\phi\left(\min _{k \in \mathcal{K}} \operatorname{SIR}_{k}(\mathbf{p}) / \gamma_{k}\right)=\min _{k \in \mathcal{K}} \phi\left(\operatorname{SIR}_{k}(\mathbf{p}) / \gamma_{k}\right)$ for every $\mathbf{p}>0$. Thus, as $\overline{\mathbf{p}} \in \mathrm{P}_{+}$,

$$
\overline{\mathbf{p}}=\underset{\mathbf{p} \in \mathrm{P}_{+}}{\arg \max } \min _{k \in \mathcal{K}} \phi\left(\operatorname{SIR}_{k}(\mathbf{p}) / \gamma_{k}\right)
$$

where $\overline{\mathbf{p}}$ is a max-min SIR-balanced power vector defined by (2). With (6) in hand, we can prove a sufficient condition for the uniqueness of $\overline{\mathbf{p}}$ under general power constraints. To this end, given $\phi$, we define the set $\mathrm{F} \subset \mathrm{Q}^{K}$ as

$$
\mathrm{F}=\left\{\mathbf{q} \in \mathrm{Q}^{K}: q_{k}=\phi\left(\operatorname{SIR}_{k}(\mathbf{p}) / \gamma_{k}\right), k \in \mathcal{K}, \mathbf{p} \in \mathrm{P}_{+}\right\}
$$

Note that $\mathrm{F}$ can be interpreted as a feasible QoS region where the QoS value for link $k$ is $\phi\left(\operatorname{SIR}_{k}(\mathbf{p}) / \gamma_{k}\right)$. By (A.2)] and [15, Sect. 5.3], the following can be said about F.

Observation 1: There is a bijective continuous map 3 from $\mathrm{F}$ onto $\mathrm{P}_{+}$(see also (23)). If

\footnotetext{
${ }^{3}$ The reader should bear in mind that the existence of such a bijective map allows us to prove some results in $\mathrm{F}$.
} 
(A.3) the inverse function $g(x):=\phi^{-1}(x)$ is log-convex [15],

then $\mathrm{F}$ is downward comprehensive, connected and convex. Moreover, $\operatorname{SIR}_{k}\left(\overline{\mathbf{p}}^{\prime}\right)$ with $\overline{\mathbf{p}}^{\prime}$ defined by (4) corresponds to a point on the boundary of $\mathrm{F}$ where $q_{1}=\ldots=q_{K}$ with $q_{k}$ defined by (7).

Note that the boundary of $\mathrm{F}$ (denoted by $\partial \mathrm{F}$ ) is the set of all points of $\mathrm{F}$ such that, if $\mathbf{p}$ is the corresponding power vector in (7), then $\mathrm{Cp} \leq \hat{\mathbf{p}}$ holds with at least one equality. Widely known examples of functions satisfying (A.2) and (A.3) are $x \mapsto \log (x), x>0$, and $x \mapsto-1 / x^{n}, n \geq 1, x>0$. Now let us state the following auxiliary result.

Lemma 1: Suppose that (A.2) and (A.3) hold. Let $\mathbf{V}$ be irreducible. Then, $\mathbf{q} \in \partial \mathrm{F}$ if and only if there exists $\mathbf{w}>0$ such that $\mathbf{q}$ maximizes $\mathbf{x} \mapsto \mathbf{w}^{T} \mathbf{x}$ over $\mathrm{F}$.

Proof: By convexity and downward comprehensivity of $\mathrm{F}$, every boundary point of this set is a maximal point and it maximizes $\mathbf{x} \mapsto \mathbf{w}^{T} \mathbf{x}$ over $\mathrm{F}$ for some $\mathbf{w} \geq 0$ [34, pp. 54-58]. Since $\mathbf{V}$ is irreducible, it follows from [35, Theorem 4.3] (see also [35, Corollary 4.3]) that $\mathbf{w}>0$.

Now we can easily observe the following.

Observation 2: If $\mathbf{V} \geq 0$ is irreducible, then $\overline{\mathbf{p}}$ is unique and equal to $\overline{\mathbf{p}}^{\prime}$ defined by (4).

Proof: The proof is deferred to Appendix VIII-A.

We complete this section by pointing out a connection between the max-min SIR-balancing problem considered in this paper and the notion of max-min fairness. A vector of balanced SIRs with entries $\operatorname{SIR}_{k}\left(\mathbf{p}^{\prime \prime}\right) / \gamma_{k}, k \in \mathcal{K}$ is max-min fair if any $\operatorname{SIR}_{k}\left(\mathbf{p}^{\prime \prime}\right) / \gamma_{k}$ cannot be increased without decreasing some $\operatorname{SIR}_{l}\left(\mathbf{p}^{\prime \prime}\right) / \gamma_{l}, l \neq k$ which is smaller than or equal to $\operatorname{SIR}_{k}\left(\mathbf{p}^{\prime \prime}\right) / \gamma_{k}$; the vector $\mathbf{p}^{\prime \prime}$ is then a max-min fair power allocation [36], [37]. A max-min fair power allocation is therefore also max-min SIR-balanced (provided that the feasible SIR region is downward comprehensive); the converse is in general not true. However, if the max-min SIR-balancing problem has a unique solution given by (4), this solution is also max-min fair and there are no other max-min fair power allocations. These relations can be observed in Fig. 1 where both max-min SIR-balanced and max-min fair points are indicated.

\section{Characterization of MaX-Min SiR-Balancing}

In this section, we characterize $\overline{\mathbf{p}} \in \mathrm{P}_{+}$defined by (2) under the assumption that $\mathrm{V}$ is irreducible. We point out possible extensions to reducible matrices at the end of this section. We assume that $\mathrm{P} \subset \mathbb{R}_{+}^{K}$ is a convex polytope given by (1). So, throughout this section, 
$\max _{n \in \mathcal{N}} g_{n}(\mathbf{p}) \leq 1$ where

$$
g_{n}(\mathbf{p}):=1 / P_{n} \mathbf{c}_{n}^{T} \mathbf{p}, \quad n \in \mathcal{N}
$$

and $\mathbf{c}_{n} \in\{0,1\}^{K}$ is a (column) vector equal to the $n$th row of the matrix C. Now, using (8), the max-min SIR power vector $\overline{\mathbf{p}}$ defined by (2) can be written as

$$
\overline{\mathbf{p}}=\underset{\mathbf{p} \geq 0}{\arg \max } \min _{k \in \mathcal{K}}\left(\operatorname{SIR}_{k}(\mathbf{p}) / \gamma_{k}\right) \quad \text { s.t. } \quad \max _{n \in \mathcal{N}} g_{n}(\mathbf{p}) \leq 1 .
$$

where $\gamma_{k}>0, k \in \mathcal{K}$, is arbitrary but fixed.

Lemma 2: Let $\overline{\mathbf{p}}$ be any power vector that solves (9). Then, the following holds

(i) $\max _{n \in \mathcal{N}} g_{n}(\overline{\mathbf{p}})=1$.

(ii) If $\mathbf{V} \geq 0$ is irreducible, then $\overline{\mathbf{p}}$ is unique and

$$
\forall_{k \in \mathcal{K}} \gamma_{k} / \operatorname{SIR}_{k}(\overline{\mathbf{p}})=\beta, \beta>0
$$

Proof: The proof can be found in Appendix VIII-B.

Because $\overline{\mathbf{p}}$ maximizes $\min _{k \in \mathcal{K}}\left(\operatorname{SIR}_{k}(\mathbf{p}) / \gamma_{k}\right)$ over $\mathrm{P}$, it follows from (10) that $1 / \beta>0$ is the corresponding maximum. It must be emphasized that (10) is not true for general nonnegative matrices $\mathbf{V} \geq 0$. In the lemma, we require that the gain matrix be irreducible, which is sufficient for (10) to hold but not necessary. The irreducibility property ensures that, regardless of the choice of $\mathrm{P}$, there is no subnetwork being completely decoupled from the rest of the network. To be more precise, if $\mathbf{V}$ is irreducible, then the network is entirely coupled by interference so that the type of power constraints is irrelevant for this issue (see also the remark at the end of this section). Unless otherwise stated, it is assumed in the remainder of this section that $\mathbf{V} \geq 0$ is an arbitrary irreducible matrix. Due to $(i i)$ of Lemma 2, this implies that the max-min SIR-balanced power vector is unique.

Let us define

$$
\mathcal{N}_{0}(\mathbf{p}):=\left\{n_{0} \in \mathcal{N}: n_{0}=\underset{n \in \mathcal{N}}{\arg \max } g_{n}(\mathbf{p})=1\right\}
$$

which includes the indices of those nodes for which the power constraints are active under the power vector p. By $(i)$ of Lemma 2, the cardinality of $\mathcal{N}_{0}(\overline{\mathbf{p}})$ must be larger than or equal to 1. In what follows, let $\beta>0$ be the constant in part $(i i)$ of the lemma. This together with part $(i)$ implies that

$$
\beta \overline{\mathbf{p}}=\boldsymbol{\Gamma V} \overline{\mathbf{p}}+\boldsymbol{\Gamma z} \quad g_{n}(\overline{\mathbf{p}})=1 .
$$


Putting the first equation into the second one yields a set of $K+1$ equations that, if written in a matrix form, show that if $\overline{\mathbf{p}}$ solves the max-min SIR-balancing problem, then there is a constant $\beta>0$ such that

$$
\beta \tilde{\mathbf{p}}=\mathbf{A}^{(n)} \tilde{\mathbf{p}}, \quad \beta>0, \tilde{\mathbf{p}} \in \mathbb{R}_{++}^{K+1}
$$

for each $n \in \mathcal{N}_{0}(\overline{\mathbf{p}})$ where $\tilde{\mathbf{p}}=(\overline{\mathbf{p}}, 1)$ is the extended power vector and the nonnegative matrix $\mathbf{A}^{(n)} \in \mathbb{R}_{+}^{(K+1) \times(K+1)}$ is defined to be

$$
\mathbf{A}^{(n)}=\left(\begin{array}{cc}
\boldsymbol{\Gamma} \mathbf{V} & \boldsymbol{\Gamma} \mathbf{z} \\
\frac{1}{P_{n}} \mathbf{c}_{n}^{T} \mathbf{\Gamma} \mathbf{V} & \frac{1}{P_{n}} \mathbf{c}_{n}^{T} \boldsymbol{\Gamma z}
\end{array}\right), \quad n \in \mathcal{N} .
$$

Alternatively, we can write (12) as $\beta \overline{\mathbf{p}}=\Gamma \mathbf{V} \overline{\mathbf{p}}+\Gamma \mathbf{z} \cdot g_{n}(\overline{\mathbf{p}})$, from which we obtain, for each $n \in \mathcal{N}_{0}(\overline{\mathbf{p}})$,

$$
\beta \overline{\mathbf{p}}=\mathbf{B}^{(n)} \overline{\mathbf{p}}, \quad \beta>0, \overline{\mathbf{p}} \in \mathbb{R}_{++}^{K+1}
$$

where $\mathbf{B}^{(n)} \in \mathbb{R}_{+}^{K \times K}$ is defined to be (for each $n \in \mathcal{N}$ )

$$
\mathbf{B}^{(n)}:=\boldsymbol{\Gamma} \mathbf{V}+\frac{1}{P_{n}} \boldsymbol{\Gamma z c}_{n}^{T}=\boldsymbol{\Gamma}\left(\mathbf{V}+\frac{1}{P_{n}} \mathbf{z c}_{n}^{T}\right)=\boldsymbol{\Gamma} \tilde{\mathbf{V}}^{(n)}
$$

and $\tilde{\mathbf{V}}^{(n)}:=\mathbf{V}+\frac{1}{P_{n}} \mathbf{z c}_{n}^{T}, n \in \mathcal{N}$. So, given $\overline{\mathbf{p}}$ defined by (9), the equations (13) and (15) hold for each $n \in \mathcal{N}_{0}(\overline{\mathbf{p}})$. In other words, the solution of (9) in a network entirely coupled by interference must satisfy (13) and (15) for each node $n \in \mathcal{N}$ whose power constraints are active at the maximum. This is summarized in the following lemma.

Lemma 3: If $\mathbf{V} \geq 0$ is irreducible and $\overline{\mathbf{p}}$ solves the max-min SIR-balancing problem (9), then $\overline{\mathbf{p}}$ satisfies both (13) and (15) for some $\beta>0$ and each $n \in \mathcal{N}_{0}(\overline{\mathbf{p}})$.

Note that the lemma is an immediate consequence of parts $(i)$ and (ii) of Lemma 2, from which (13) and (15) follow for an arbitrary $n \in \mathcal{N}_{0}(\overline{\mathbf{p}})$. Now we are in a position to prove the following result.

Lemma 4: Suppose that $\mathbf{V} \geq 0$ is irreducible. Then, for any constants $c_{1}>0$ and $c_{2}>0$, the following holds.

(i) For each $n \in \mathcal{N}$, there is exactly one positive vector $\mathbf{p}=\mathbf{p}^{(n)} \in \mathbb{R}_{+}^{K}$ with $g_{n}(\mathbf{p})=c_{1}$ satisfying $\beta^{(n)} \mathbf{p}=\mathbf{B}^{(n)} \mathbf{p}$ for some $\beta^{(n)}>0$. Moreover, $\beta^{(n)}$ is a simple eigenvalue of $\mathbf{B}^{(n)}$ and $\beta^{(n)}=\rho\left(\mathbf{B}^{(n)}\right)$. 
(ii) For each $n \in \mathcal{N}$, there is exactly one positive vector $\tilde{\mathbf{p}}=\tilde{\mathbf{p}}^{(n)} \in \mathbb{R}_{+}^{K+1}$ with $\tilde{p}_{K+1}=c_{2}$ satisfying $\beta^{(n)} \tilde{\mathbf{p}}=\mathbf{A}^{(n)} \tilde{\mathbf{p}}$ for some $\beta^{(n)}>0$. Moreover, $\beta^{(n)}$ is a simple eigenvalue of $\mathbf{A}^{(n)}$ and $\beta^{(n)}=\rho\left(\mathbf{A}^{(n)}\right)$.

Proof: The reader can find the proof in Appendix VIII-C,

The lemma says that, for each $n \in \mathcal{N}$, the matrix equation $\beta^{(n)} \mathbf{p}=\mathbf{B}^{(n)} \mathbf{p}$ with $\beta^{(n)}>0$ and $\mathbf{p} \in \mathbb{R}_{+}^{K}$ is satisfied if and only if $\mathbf{p}$ is a positive right eigenvector of $\mathbf{B}^{(n)}$ associated with $\beta^{(n)}=\rho\left(\mathbf{B}^{(n)}\right)$. Furthermore, if $g_{n}(\mathbf{p})=1$, then $\mathbf{p}$ is unique. Similarly, $\beta^{(n)} \mathbf{p}=\mathbf{A}^{(n)} \mathbf{p}$ with $\beta^{(n)}>0$ and $\mathbf{p} \in \mathbb{R}_{+}^{K+1}$ is satisfied if and only if $\mathbf{p}$ is a positive right eigenvector of $\mathbf{A}^{(n)}$ associated with $\beta^{(n)}=\rho\left(\mathbf{A}^{(n)}\right)$ and there is exactly one such an eigenvector whose last entry is equal to one. Furthermore, for each $n \in \mathcal{N}, \beta^{(n)}=\rho\left(\mathbf{A}^{(n)}\right)=\rho\left(\mathbf{B}^{(n)}\right)$. This is because if $\rho\left(\mathbf{B}^{(n)}\right) \mathbf{p}=\mathbf{B}^{(n)} \mathbf{p}$ holds for some $n \in \mathcal{N}$, then we must have $\rho\left(\mathbf{B}^{(n)}\right) \tilde{\mathbf{p}}=\mathbf{A}^{(n)} \tilde{\mathbf{p}}$ where $\tilde{\mathbf{p}}=(\mathbf{p}, 1) \in \mathbb{R}_{++}^{K}$. Thus, by Lemma 4, we must have

$$
\rho\left(\mathbf{A}^{(n)}\right)=\rho\left(\mathbf{B}^{(n)}\right), \quad n \in \mathcal{N}
$$

Note that a solution to the max-min SIR-balancing problem is not necessarily obtained for each $n \in \mathcal{N}$ since, in the optimum, some power constraints may be inactive. Indeed, in general, the set $\mathcal{N}_{0}^{c}(\overline{\mathbf{p}})=\mathcal{N} \backslash \mathcal{N}_{0}(\overline{\mathbf{p}})$ is not an empty set where $\overline{\mathbf{p}}$ defined by (9) is unique due to (ii) of Lemma 2 .

Now we combine Lemmas 3 and 4 to obtain the following.

Theorem 1: Let $\beta$ be given by (10). If $\mathrm{V} \geq 0$ is irreducible, then the following statements are equivalent.

(i) $\overline{\mathbf{p}} \in \mathrm{P}_{+}$solves the max-min SIR-balancing problem (9).

(ii) For each $n \in \mathcal{N}_{0}(\overline{\mathbf{p}}), \overline{\mathbf{p}}$ is a unique positive right eigenvector of $\mathbf{B}^{(n)}$ associated with $\beta=\rho\left(\mathbf{B}^{(n)}\right)>0$ such that $g_{n}(\overline{\mathbf{p}})=1$.

(iii) For each $n \in \mathcal{N}_{0}(\overline{\mathbf{p}}), \tilde{\mathbf{p}}$ is a unique positive right eigenvector of $\mathbf{A}^{(n)}$ associated with $\beta=\rho\left(\mathbf{A}^{(n)}\right)>0$ such that $\tilde{p}_{K+1}=1$.

Proof: The proof can be found in Appendix VIII-D,

Theorem 1 implies that if $\mathbf{V}$ is irreducible, then $\overline{\mathbf{p}}>0$ is the (positive) right eigenvector of $\mathbf{B}^{(n)}$ associated with $\rho\left(\mathbf{B}^{(n)}\right) \in \sigma\left(\mathbf{B}^{(n)}\right)$ for each $n \in \mathcal{N}_{0}(\overline{\mathbf{p}})$. Alternatively, $\overline{\mathbf{p}}$ can be obtained from $\tilde{\mathbf{p}}=(\overline{\mathbf{p}}, 1)$, which is the positive right eigenvector of $\mathbf{A}^{(n)}$ associated with $\rho\left(\mathbf{A}^{(n)}\right)$ for each $n \in \mathcal{N}_{0}(\overline{\mathbf{p}})$. The problem is, however, that $\mathcal{N}_{0}(\overline{\mathbf{p}})$ is not known as this set is determined by the 
solution to the max-min SIR-balancing problem, and hence its determination is itself a part of the problem. As the SIR targets are feasible if and only if they are met under $\bar{p}$, the following characterization of the set $\mathcal{N}_{0}(\overline{\mathbf{p}})$ immediately follows from [28] and (17).

Theorem 2 ([28]): Suppose that $\mathbf{V} \geq 0$ is irreducible. Then,

$$
\mathcal{N}_{0}(\overline{\mathbf{p}})=\left\{n_{0} \in \mathcal{N}: n_{0}=\arg \max _{n \in \mathcal{N}} \rho\left(\mathbf{B}^{(n)}\right)\right\}
$$

Moreover, the feasible SIR region $\mathrm{F}_{\gamma}$ is characterized as

$$
\begin{gathered}
\mathrm{F}_{\gamma}=\left\{\boldsymbol{\gamma} \in \mathbb{R}_{++}^{K}: \max _{n \in \mathcal{N}} \rho\left(\mathbf{B}^{(n)}\right)=\max _{n \in \mathcal{N}} \rho\left(\mathbf{A}^{(n)}\right) \leq 1,\right. \\
\left.\Gamma=\operatorname{diag}\left(\gamma_{1}, \ldots, \gamma_{K}\right)\right\} .
\end{gathered}
$$

The characterization of the feasible SIR region in (19) can be deduced directly from [28] as the authors show that $\min _{k \in \mathcal{K}}\left(\operatorname{SIR}_{k}(\mathbf{p}) / \gamma_{k}\right) \leq \min _{n \in \mathcal{N}}\left(1 / \rho\left(\mathbf{B}^{n}\right)\right)$, without characterizing, however, the corresponding power allocation vector $\mathbf{p}$.

Theorems 1 and 2 directly lead to the following procedure for computing the max-min SIR power vector $\overline{\mathbf{p}}$ given by (2).

Input: $\boldsymbol{\Gamma}=\operatorname{diag}\left(\gamma_{1}, \ldots, \gamma_{K}\right)$.

Output: $\overline{\mathbf{p}} \in \mathrm{P}_{+} \subset \mathrm{P}$

1: Find an arbitrary index $n_{0} \in \mathcal{N}$ such that $n_{0}=\arg \max _{n \in \mathcal{N}} \rho\left(\mathbf{B}^{(n)}\right)$ where $\mathbf{B}^{(n)}$ is given by (16).

2: Let $\overline{\mathbf{p}}$ be given by $\rho\left(\mathbf{B}^{\left(n_{0}\right)}\right) \overline{\mathbf{p}}=\mathbf{B}^{\left(n_{0}\right)} \overline{\mathbf{p}}$ and normalized such that $\mathbf{c}_{n_{0}}^{T} \overline{\mathbf{p}}=P_{n_{0}}$.

Remark 1 (Remark on reducible matrices): We point out that all the statements in this paper hold if $\mathbf{B}^{(n)}$ is irreducible for each $n \in \mathcal{N}$, which may be satisfied even if $\mathbf{V}$ is reducible. This is for instance true in the case of a sum power constraint $(\mathbf{C}=(1, \ldots, 1))$ where $\mathbf{B}=\mathbf{B}^{(1)}, \mathcal{N}=$ $\{1\}$, is a positive matrix. Moreover, the statement of Theorem 1 can be shown to be true even if $\mathbf{B}^{(n)}$ is irreducible only for $n \in \mathcal{N}_{0}(\overline{\mathbf{p}})$.

\section{Applications}

In this section, we discuss two other applications of the results. In doing so, $\mathrm{V}$ is assumed to be irreducible. Under this assumption, the feasible QoS region given by (7) can be shown to be strictly convex [35, Corrolary 4.3]. 


\section{A. Computation via utility-based power control}

In this section, we show that $\overline{\mathbf{p}}$ can be obtained by maximizing the following aggregate utility function

$$
F(\mathbf{p}, \mathbf{w}):=\sum_{k \in \mathcal{K}} w_{k} \phi\left(\operatorname{SIR}_{k}(\mathbf{p}) / \gamma_{k}\right)
$$

provided that the weight vector $\mathbf{w}=\left(w_{1}, \ldots, w_{K}\right)>0$ is chosen appropriately and $\phi: \mathbb{R}_{++} \rightarrow \mathrm{Q}$ satisfies (A.2) and (A.3), To this end, define

$$
\mathbf{p}^{*}(\mathbf{w}):=\arg \max _{\mathbf{p} \in \mathrm{P}_{+}} F(\mathbf{p}, \mathbf{w}) .
$$

Although $\mathrm{P}_{+}$is not compact, it can be shown [15] that the maximum exists if (A.2) and (A.3) are fulfilled. Furthermore, it is obvious that in the maximum, at least one power constraint is active, that is, $\mathbf{C p}^{*} \leq \hat{\mathbf{p}}$ holds at least with one equality. Thus, we have $\mathbf{q}^{*}(\mathbf{w})=\left(q_{1}^{*}(\mathbf{w}), \ldots, q_{K}^{*}(\mathbf{w})\right) \in$ $\partial \mathrm{F}$ for

$$
q_{k}^{*}(\mathbf{w})=\phi\left(\operatorname{SIR}_{k}\left(\mathbf{p}^{*}(\mathbf{w})\right) / \gamma_{k}\right) .
$$

In words, $\mathbf{p}^{*}=\mathbf{p}^{*}(\mathbf{w})$ corresponds to a boundary point of $\mathrm{F}$ defined by (7). Different boundary points can be achieved by choosing different weight vectors in (20). In particular, Lemma 1 implies that $\mathbf{q} \in \partial \mathrm{F}$ if and only if $\mathbf{q}=\mathbf{q}^{*}(\mathbf{w})$ for some $\mathbf{w}>0$. For the analysis in this section, it is important to recall from Observation 1 that there is a bijective map from $\mathrm{F}$ onto $\mathrm{P}$ and this map can be shown to be [15, Section 5.3]

$$
\mathbf{p}(\mathbf{q})=(\mathbf{I}-\mathbf{G}(\mathbf{q}) \mathbf{\Gamma V})^{-1} \mathbf{G}(\mathbf{q}) \mathbf{\Gamma} \mathbf{z}, \mathbf{q} \in \mathrm{F}
$$

where $\mathbf{G}(\mathbf{q}):=\operatorname{diag}\left(g\left(q_{1}\right), \ldots, g\left(q_{K}\right)\right)$ with $g(x)$ defined by (A.3) and $\rho(\mathbf{G}(\mathbf{q}) \mathbf{\Gamma V})<1$, which ensures the existence of $\mathbf{p}(\mathbf{q})$ and is satisfied for every $\mathbf{q} \in \mathrm{F}$ [15, Section 5.3]. Note that $g$ is strictly increasing and $g(x)>0$ for all $x \in \mathrm{Q}$, which follows from (A.2) and (A.3),

Lemma 5: $\mathbf{q} \in \partial \mathrm{F}$ if and only if $\max _{n \in \mathcal{N}} \lambda_{n}(\mathbf{q})=1$ where $\lambda_{n}(\mathbf{q}):=\rho\left(\mathbf{G}(\mathbf{q}) \mathbf{B}^{(n)}\right)>$ $\rho(\mathbf{G}(\mathbf{q}) \mathbf{\Gamma V})>0$.

Proof: The proof is deferred to Appendix VIII-E

Now we are in a position to prove the following.

Theorem 3: Suppose that (A.2) and (A.3) hold. Let $\mathbf{q} \in \partial \mathrm{F}$ and $\mathbf{u}(\mathbf{q})=\left(g^{\prime}\left(q_{1}\right) / g\left(q_{1}\right), \ldots, g^{\prime}\left(q_{K}\right) / g\left(q_{K}\right)\right)>$ 0 . Then, we have $\mathbf{q}=\mathbf{q}^{*}(\mathbf{w})$ given by (22) if

$$
\mathbf{w}=c \cdot \mathbf{u}(\mathbf{q}) \circ \mathbf{y} \circ \mathbf{x}, \quad c>0
$$


where $y$ and $\mathrm{x}$ are positive left and right eigenvectors of $\mathbf{G}(\mathbf{q}) \mathbf{B}^{\left(n_{0}\right)}$, respectively, associated with $\lambda_{n_{0}}(\mathbf{q})$ for any $n_{0}=\arg \max _{n \in \mathcal{N}} \lambda_{n}(\mathbf{q})$.

Proof: The reader can find the proof in Appendix VIII-F.

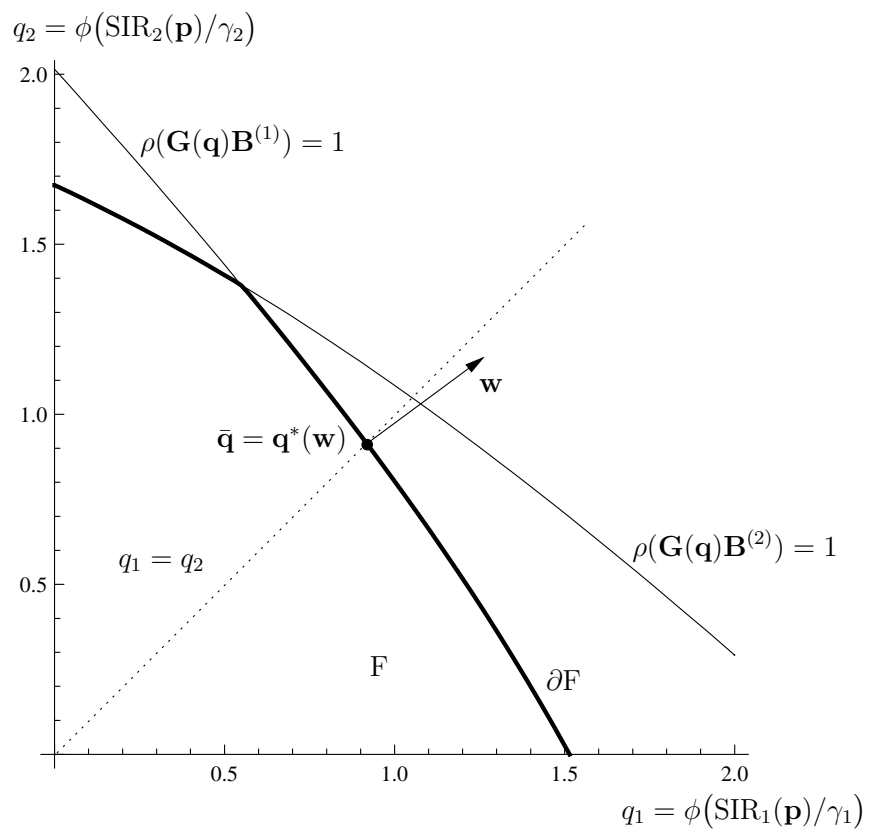

Fig. 2. An illustration of Corollary 1 The figure shows an example of the feasible QoS region defined by (7) with 2 users subject to individual power constraints $(\mathbf{C}=\mathbf{I})$. The point $\overline{\mathbf{q}}=\mathbf{q}^{*}(\mathbf{w})$ with $\mathbf{w}=c \mathbf{y} \circ \mathbf{x}, c>0$, corresponds to the unique max-min SIR-balanced power allocation. The weight vector $\mathbf{w}$ is normal to a hyperplane which supports the feasible QoS region at $\overline{\mathbf{q}} \in \partial \mathrm{F}$.

Now we can establish a connection between (2) and (21). The connection is illustrated by Figure 2 ,

Corollary 1: Let $\mathbf{y}$ and $\mathbf{x}$ be positive left and right eigenvectors of $\mathbf{B}^{\left(n_{0}\right)}$ associated with $\rho\left(\mathbf{B}^{\left(n_{0}\right)}\right)$ for any $n_{0} \in \mathcal{N}_{0}(\overline{\mathbf{p}})$. If $\mathbf{w}=c \mathbf{y} \circ \mathbf{x}, c>0$, then $\overline{\mathbf{p}}=\mathbf{p}^{*}(\mathbf{w})$.

Proof: The proof can be found in Appendix VIII-G.

\section{B. Saddle point characterization of the Perron roots}

Finally, we point out that Theorem 1 gives rise to a saddle point characterization of $\rho\left(\mathbf{B}^{(n)}\right), n \in$ $\mathcal{N}_{0}(\overline{\mathbf{p}})$. Let $\Pi_{K}:=\left\{\mathbf{x} \in \mathbb{R}_{+}^{K}:\|\mathbf{x}\|_{1}=1\right\}$ and $\Pi_{K}^{+}=\Pi_{K} \cap \mathbb{R}_{++}^{K}$. We define $G: \Pi_{K}^{+} \times \mathrm{P}_{+} \rightarrow \mathbb{R}$ as

$$
G(\mathbf{w}, \mathbf{p}):=\sum_{k \in \mathcal{K}} w_{k} \psi\left(\gamma_{k} / \operatorname{SIR}_{k}(\mathbf{p})\right)
$$


where $\psi(x):=-\phi(1 / x)$. A key ingredient in the proof of the saddle point characterization is the following theorem, which can be deduced from [15, Sections 1.2.4-5]:

Theorem 4: Assume that (A.1) (A.3) hold and $\mathbf{V}$ is irreducible. Let $\mathbf{B}=\mathbf{B}^{(n)}$ for any $n \in \mathcal{N}$, and let $\mathbf{w}=\mathbf{y} \circ \mathbf{x} \in \Pi_{K}^{+}$where $\mathbf{y}$ and $\mathbf{x}$ are positive left and right eigenvectors of $\mathbf{B}$ associated with $\rho(\mathbf{B})$. Then, for all $\mathbf{p}>0$,

$$
\psi(\rho(\mathbf{B})) \leq \sum_{k \in \mathcal{K}} w_{k} \psi\left(\frac{(\mathbf{B} \mathbf{p})_{k}}{p_{k}}\right) .
$$

Equality holds if and only if $\mathbf{p}=\mathbf{x}>0$.

Now we are in a position to present the saddle point characterization of the Perron root of $\mathbf{B}^{(n)}, n \in \mathcal{N}_{0}(\overline{\mathbf{p}})$, similar to the one for the noiseless case which can be found in [15].

Theorem 5: Suppose that (A.2) and (A.3) hold, and V is irreducible. Then,

$$
\psi\left(\rho\left(\mathbf{B}^{\left(n_{0}\right)}\right)\right)=\sup _{\mathbf{w} \in \Pi_{K}^{+}} \inf _{\mathbf{p} \in \mathrm{P}_{+}} G(\mathbf{w}, \mathbf{p})=\inf _{\mathbf{p} \in \mathrm{P}_{+}} \sup _{\mathbf{w} \in \Pi_{K}^{+}} G(\mathbf{w}, \mathbf{p})
$$

where $n_{0}=\arg \max _{n \in \mathcal{N}} \rho\left(\mathbf{B}^{(n)}\right)$. Moreover, a point $\left(\mathbf{w}^{*}, \mathbf{p}^{*}\right)$ is the saddle point of $G(\mathbf{w}, \mathbf{p})$ if and only if $\mathbf{p}^{*}=\overline{\mathbf{p}}$ and $\mathbf{w} \in \mathrm{W}$, where

$$
\mathrm{W}=\left\{\mathbf{w} \in \Pi_{K}^{+}: \mathbf{w}=\sum_{n \in \mathcal{N}_{0}} c_{n} \mathbf{w}^{(n)}, \sum_{n \in \mathcal{N}_{0}} c_{n}=1, c_{n} \geq 0\right\}
$$

and $\mathbf{w}^{(n)}:=\mathbf{y}^{(n)} \circ \mathbf{x}^{(n)} \in \Pi_{K}^{+}$with $\rho\left(\mathbf{B}^{(n)}\right) \mathbf{x}^{(n)}=\mathbf{B}^{(n)} \mathbf{x}^{(n)}, \rho\left(\mathbf{B}^{(n)}\right) \mathbf{y}^{(n)}=\left(\mathbf{B}^{(n)}\right)^{T} \mathbf{y}^{(n)}$, $\left(\mathbf{y}^{(n)}\right)^{T} \mathbf{X}^{(n)}=1$. In words, at the saddle point the power vector is equal to the max-min SIRbalanced power allocation, whereas the weight vector is any linear combination of the vectors $\mathbf{w}^{(n)}$ for $n \in \mathcal{N}_{0}(\overline{\mathbf{p}})$.

With Theorems 11 and 4 in hand, the proof is similar to that in [15, Section 1.2.4]. The existence of a saddle point is ensured by irreducibility of the gain matrix since then positive left and right eigenvectors exist. The uniqueness follows from the irreducibility property and the normalizations.

The reason why the theorem is of interest is that it provides a basis for the design of alternative power control algorithms for saddle point problems that converge to $\overline{\mathbf{p}}$ and may be amenable to distributed implementation. Basically, the idea of the algorithm is redolent of that of primal-dual algorithms that converge to a saddle point of the associated Lagrange function [34]. Development of new algorithms is currently a subject of our ongoing work; the main idea, however, consists in minimizing the function $G(\mathbf{w}, \mathbf{p})$ with respect to $\mathbf{p}$, and simultaneously maximizing $G(\mathbf{w}, \mathbf{p})$ 
with respect to $\mathrm{w}$. The straight-forward approach employs the gradient projection method, where in order for the objective function to be convex in the power variable the substitution $\mathbf{s}=\log (\mathbf{p})$ is used. Each iteration encompasses the calculation of the gradient of $G\left(\mathbf{w}, e^{\mathbf{s}}\right)$, and an update of the vectors $\mathrm{w}$ and $\mathrm{s}$ in the direction and against the direction of the gradient, respectively. This process requires a suitable step size to be chosen. The iteration is concluded with a projection of the updated values of $\mathrm{w}$ and $\mathrm{s}$ onto the corresponding sets of valid values.

The minimization of $G(\mathbf{w}, \mathbf{p})$ in the power domain using the gradient projection method corresponds to employing the power control algorithm presented in [15, Section 6.5]. In particular, the gradient can be computed in a distributed way, and the projection onto the feasible set is also distributedly implementable in many cases of interest. As for the optimization in the weights domain, the gradient can be computed independently by each node, but performing the projection requires in general centralized operation.

\section{CONCLuSions}

In this paper, we have characterized the max-min SIR-balanced power allocation under general power constraints. This characterization is an extension of the results known previously for the noiseless case, in which the power constraints play no role. We have also established a connection between the max-min SIR-balancing power allocation problem and the utility-based power allocation problem for the considered case, and as an application of our results we have discussed two classes of power allocation algorithms based on those two approaches. Finally, we have presented a saddle-point characterization of the Perron roots of the extended gain matrices which may constitute a basis for developing distributed power control algorithms.

\section{ACKNOWLEDGMENTS}

We thank our colleagues Dr. Chee Wei Tan, Prof. Mung Chiang and Prof. R. Srikant for bringing to our attention their Infocom'09 paper [38], after the publication of our technical report [39] on which the present paper is fully based. In their paper [38], they have independently obtained some related results. We also refer the reader to [40] for additional views on the issues.

Finally, as explained in [15], Theorem 4 of this paper is substantially related to results of the seminal paper of Friedland \& Karlin [41] to which also the reader is referred. 


\section{APPENDIX}

\section{A. Proof of Observation 2}

Let (A.3) be satisfied, and let $\overline{\mathbf{p}} \in \mathrm{P}_{+}$be any solution to (2) or, equivalently, (6). Let $\bar{q}_{k}=$ $\phi\left(\operatorname{SIR}_{k}(\overline{\mathbf{p}}) / \gamma_{k}\right), k \in \mathcal{K}$. By Observation 1, $\mathrm{F}$ is a convex downward comprehensive set and, by (A.2), (6) and (7), $\overline{\mathbf{q}}=\left(\bar{q}_{1}, \ldots, \bar{q}_{K}\right) \in \partial \mathrm{F}$ is its boundary point since at least one power constraint is active in the optimum (see Lemma 2). Thus, by irreducibility of $\mathbf{V}$ and Lemma 1. there exists $\mathbf{w}>0$ such that $\mathbf{w}^{T}(\overline{\mathbf{q}}-\mathbf{u}) \geq 0$ for all $\mathbf{u} \in \mathrm{F}$. Due to positivity of $\mathbf{w}$, this implies that for any $\mathbf{u} \in \mathrm{F}, \mathbf{u} \neq \overline{\mathbf{q}}$, there is at least one index $i=i(\overline{\mathbf{q}}, \mathbf{u}) \in \mathcal{K}$ such that $\bar{q}_{i}>u_{i}$. In particular, for any $\mathbf{u} \in \mathrm{F}, \mathbf{u} \neq \overline{\mathbf{q}}^{\prime}$ there is $i=i\left(\overline{\mathbf{q}}^{\prime}, \mathbf{u}\right) \in \mathcal{K}$ such that $\bar{q}_{i}^{\prime}>u_{i}$ where $\bar{q}_{k}^{\prime}=\phi\left(\operatorname{SIR}_{k}\left(\overline{\mathbf{p}}^{\prime}\right) / \gamma_{k}\right), k \in \mathcal{K}$. On the other hand, however, we have $\overline{\mathbf{q}}^{\prime} \leq \overline{\mathbf{q}}$. This is simply because $\bar{q}_{1}^{\prime}=\cdots=\bar{q}_{K}^{\prime}=\min _{k \in \mathcal{K}} \phi\left(\operatorname{SIR}_{k}(\overline{\mathbf{p}})\right)$. Combining both inequalities shows that $\overline{\mathbf{q}}=\overline{\mathbf{q}}^{\prime}$, and hence, by bijectivity, we obtain $\overline{\mathbf{p}}=\overline{\mathbf{p}}^{\prime}$, which is unique by [15, Theorem A.51].

\section{B. Proof of Lemma 2}

Part (i) should be obvious since if we had $g_{n}(\overline{\mathbf{p}})<1$ for all $n \in \mathcal{N}$, then it would be possible to increase $\min _{k \in \mathcal{K}} \operatorname{SIR}_{k}(\overline{\mathbf{p}}) / \gamma_{k}$ by allocating the power vector $c \overline{\mathbf{p}} \in \mathrm{P}_{+}$with $c=$ $1 / \max _{n \in \mathcal{N}} g_{n}(\overline{\mathbf{p}})>1$. In order to show part $(i i)$, note that if (A.2) and (A.3) hold, then, by Observation 1, $\mathrm{F}$ is a convex downward comprehensive set. Moreover, $\overline{\mathbf{p}} \in \mathrm{P}_{+}$given by (6) corresponds to a point $\overline{\mathbf{q}} \in \partial \mathrm{F}$, with $\bar{q}_{k}=\phi\left(\operatorname{SIR}_{k}(\overline{\mathbf{p}}) / \gamma_{k}\right), k \in \mathcal{K}$. Thus, by irreducibility of $\mathbf{V}$, it follows from Lemma 1 that $\overline{\mathbf{q}}$ is a maximal point of $\mathrm{F}$, and hence $\overline{\mathbf{q}} \leq \mathbf{q}$ for any $\mathbf{q} \in \mathrm{F}$ implies that $\mathbf{q}=\overline{\mathbf{q}}$ [34]. That is, there is no vector in $\mathrm{F}$ that is larger in all components than $\overline{\mathbf{q}}$. On the other hand, by the discussion in Sect. III, $\overline{\mathbf{q}}$ is a point where the hyperplane in the direction of the vector $(1 / K, \ldots, 1 / K)$ intersects the boundary of $\mathrm{F}$. As a result, $\bar{q}_{1}=\cdots=\bar{q}_{K}$, which together with the maximality property and strict monotonicity of $\phi$, shows that $\operatorname{SIR}_{k}(\overline{\mathbf{p}}) / \gamma_{k}=\beta$ for each $k \in \mathcal{K}$ where $\beta$ is positive due to $(i)$. If $\mathbf{V}$ is irreducible, the uniqueness of $\overline{\mathbf{p}}$ follows from Observation 2

\section{Proof of Lemma 4}

Let $n \in \mathcal{N}$ be arbitrary. First we prove part $(i)$. Since $1 / P_{n} \mathbf{z c}_{n}^{T} \geq 0$ and $\mathbf{V}$ is irreducible, we can conclude from (16) that $\mathbf{B}^{(n)} \geq 0$ is irreducible as well. Thus, by the Perron-Frobenius 
theorem for irreducible matrices [33], [32], there exists a positive vector $\mathbf{p}$ which is an eigenvector of $\mathbf{B}^{(n)}$ associated with $\rho\left(\mathbf{B}^{(n)}\right)$, and there are no nonnegative eigenvectors of $\mathbf{B}^{(n)}$ associated with $\rho\left(\mathbf{B}^{(n)}\right)$ other than $\mathbf{p}$ and its positive multiples. Among all the positive eigenvectors, there is exactly one eigenvector $\mathbf{p}>0$ such that $g_{n}(\mathbf{p})=c_{1}$. This proves part $(i)$. In order to prove (ii), note that if $\mathbf{A}^{(n)}$ was irreducible, then we could invoke the Perron-Frobenius theorem and proceed essentially as in part $(i)$ to conclude $(i i)$ (with the uniqueness property resulting from the normalization of the eigenvector so that its last component is equal to $c_{2}>0$ ). In order to show that $\mathbf{A}^{(n)}$ is irreducible, let $\mathcal{G}\left(\mathbf{A}^{(n)}\right)$ be the associated directed graph of $\{1, \ldots, K+1\}$ nodes [33]. Since $\Gamma \mathrm{V}$ is irreducible, it follows that the subgraph $\mathcal{G}(\boldsymbol{\Gamma V})$ is strongly connected [33]. Furthermore, as the vector $\Gamma \mathrm{z}$ is positive, we can conclude from (14) that there is a directed edge leading from node $K+1$ to each node $n<K+1$ belonging to the subgraph $\mathcal{G}(\boldsymbol{\Gamma V})$. Finally, note that as $\Gamma \mathrm{V}$ is irreducible, each row of $\Gamma \mathrm{V}$ has at least one positive entry. Hence, the vector $1 / P_{n} \mathbf{c}_{n}^{T} \boldsymbol{\Gamma V}$ has at least one positive entry as well, from which and (14) it follows that there is a directed edge leading from a node belonging to $\mathcal{G}(\boldsymbol{\Gamma V})$ to node $K+1$. So, $\mathcal{G}\left(\mathbf{A}^{(n)}\right)$ is strongly connected, and thus $\mathbf{A}^{(n)}$ is irreducible.

\section{Proof of Theorem 1}

$(i) \rightarrow($ ii $)$ : By Lemma $3 \overline{\mathbf{p}} \in \mathrm{P}_{+}$satisfies (15) for some $\beta>0$. Thus, by Lemma 4 part $(i)$ implies part (ii). (ii) $\rightarrow$ (iii): Given any $n \in \mathcal{N}_{0}(\overline{\mathbf{p}})$, it follows from (15) that $\rho\left(\mathbf{B}^{(n)}\right) \overline{\mathbf{p}}=\mathbf{B}^{(n)} \overline{\mathbf{p}}$ with $g_{n}(\overline{\mathbf{p}})=1$ is equivalent to $\rho\left(\mathbf{B}^{(n)}\right) \overline{\mathbf{p}}=\Gamma \mathbf{V} \overline{\mathbf{p}}+\Gamma \mathbf{z}$, which in turn can be rewritten to give (13) with $\beta=\rho\left(\mathbf{B}^{(n)}\right)$ and $\tilde{\mathbf{p}}=(\overline{\mathbf{p}}, 1)$. Since $\overline{\mathbf{p}}$ is positive, so is also $\tilde{\mathbf{p}}$. Thus, $\tilde{\mathbf{p}}$ with $\tilde{p}_{K+1}=1$ is a positive right eigenvector of $\mathbf{A}^{(n)}$ and the associated eigenvalue is equal to $\rho\left(\mathbf{B}^{(n)}\right)>0$. So, considering part (iii) of Lemma 4 we can conclude that (iii) follows from (ii). (iii) $\rightarrow($ i): By Lemma 4 for each $n \in \mathcal{N}_{0}(\overline{\mathbf{p}})$, there exists exactly one positive vector $\tilde{\mathbf{p}}$ with $\tilde{p}_{K+1}=1$ such that (13) is satisfied. Furthermore, $\beta=\rho\left(\mathbf{A}^{(n)}\right), n \in \mathcal{N}_{0}(\overline{\mathbf{p}})$ is a simple eigenvalue of $\mathbf{A}^{(n)}$. Now considering Lemma 3 proves the last missing implication.

\section{E. Proof of Lemma 5}

By (7) with (A.2), we have $\mathbf{q} \in \mathrm{F}$ if and only if there is $\mathbf{p} \in \mathrm{P}$ such that $\phi\left(\operatorname{SIR}_{k}(\mathbf{p}) / \gamma_{k}\right) \geq q_{k}$ for each $k \in \mathcal{K}$. Thus, $\mathbf{q} \in \mathrm{F}$ if and only if $1 / \lambda:=\max _{\mathbf{p} \in \mathrm{P}} \min _{k \in \mathcal{K}}\left(\operatorname{SIR}_{k}(\mathbf{p}) / \gamma_{k} g\left(q_{k}\right)\right) \geq 1$ where the maximum always exists. Comparing the left hand side of the inequality above with 
(2) shows that the only difference to the original problem formulation is that $\gamma_{k}$ is substituted by $\gamma_{k} g\left(q_{k}\right)$ or, equivalently, $\boldsymbol{\Gamma}$ by $\mathbf{G}(\mathbf{q}) \boldsymbol{\Gamma}$, which is positive definite as well. Thus, by (10), Theorem 11 and Theorem 2, we have $\mathbf{q} \in \mathrm{F}$ if and only if $\lambda=\max _{n \in \mathcal{N}} \lambda_{n}(\mathbf{q}) \leq 1$. Moreover, $\mathbf{p}(\mathbf{q})$ given by (23) is the unique power vector such that $q_{k}=\phi\left(\operatorname{SIR}_{k}(\mathbf{p}(\mathbf{q})) / \gamma_{k}\right)$ for each $k \in \mathcal{K}$. Since the Neumann series converges for any $\mathbf{q} \in \mathrm{F}$, we have $\mathbf{p}(\mathbf{q})=\sum_{l=0}^{\infty}(\mathbf{G}(\mathbf{q}) \boldsymbol{\Gamma V})^{l} \mathbf{G}(\mathbf{q}) \boldsymbol{\Gamma} \mathbf{z}$. Now as $\mathbf{G}(\mathbf{q}) \Gamma z$ is positive and $\mathbf{G}(\mathbf{q}) \Gamma \mathrm{V}$ is irreducible, we can conclude from [15, Lemma A.28] and (A.2) that each entry of $\mathbf{p}(\mathbf{q})$ is strictly increasing in each entry of $\mathbf{q}$. Thus, as $\mathrm{F}$ is downward comprehensive and $\mathbf{q} \notin \partial \mathrm{F}$ holds if and only if all power constraints are inactive, for every $\mathbf{q} \in \operatorname{int}(\mathrm{F})$, there is $\tilde{\mathbf{q}} \in \partial \mathrm{F}$ such that $\tilde{\mathbf{q}}=\mathbf{q}+\mathbf{u}$ for some $\mathbf{u}>0$. By irreducibility of $\mathbf{B}^{(n)}$, this implies that $\lambda_{n}(\mathbf{q})<\lambda_{n}(\mathbf{q}+\mathbf{u})=\lambda_{n}(\tilde{\mathbf{q}}) \leq 1$ for each $n \in \mathcal{N}$ So, if $\max _{n \in \mathcal{N}} \lambda_{n}(\mathbf{q})=1$, then $\mathbf{q} \in \partial \mathrm{F}$. Conversely, if $\mathbf{q} \in \partial \mathrm{F}$, we must have $\max _{n \in \mathcal{N}} \lambda_{n}(\mathbf{q})=1$ since otherwise there would exist $\tilde{\mathbf{q}} \notin \mathrm{F}$ such that $\max _{n \in \mathcal{N}} \lambda_{n}(\tilde{\mathbf{q}})=1$, which would contradict Theorem 2 . This completes the proof.

\section{F. Proof of Theorem 3}

Let $\tilde{\mathbf{q}} \in \partial \mathrm{F}$ and $n_{0}=\arg \max _{n \in \mathcal{N}} \lambda_{n}(\mathbf{q})$ be arbitrary and note that $\mathrm{F}$ is a convex set. So, by Lemma 1, there is $\mathbf{w}>0$ such that $\tilde{\mathbf{q}}$ maximizes $\mathbf{q} \mapsto \mathbf{w}^{T} \mathbf{q}$ over F. Lemma 5 implies that this convex problem can be stated as $\tilde{\mathbf{q}}=\arg \max _{\mathbf{q}} \mathbf{w}^{T} \mathbf{q}$ subject to $\lambda_{n_{0}}(\mathbf{q})=1, \mathbf{q} \in \mathrm{Q}^{K}$. Due to (A.2), the spectral radius is continuously differentiable on $\mathrm{Q}^{K}$. Thus, the Karush-Kuhn-Tucker conditions [34], which are necessary and sufficient for optimality (due to the convexity property), imply that $\mathbf{w}$ is parallel with $\nabla \lambda_{n_{0}}(\mathbf{q})$. Now, by [42], we have $\frac{\partial \lambda_{n_{0}}(\mathbf{q})}{\partial q_{k}}=y_{k} g^{\prime}\left(q_{k}\right) \sum_{l \in \mathcal{K}} b_{k, l}^{\left(n_{0}\right)} x_{l}=$ $\frac{g^{\prime}\left(q_{k}\right)}{g\left(q_{k}\right)} y_{k} \sum_{l \in \mathcal{K}} g\left(q_{k}\right) b_{k, l}^{\left(n_{0}\right)} x_{l}=\lambda_{n_{0}}(\mathbf{q}) \frac{g^{\prime}\left(q_{k}\right)}{g\left(q_{k}\right)} y_{k} x_{k}=\frac{g^{\prime}\left(q_{k}\right)}{g\left(q_{k}\right)} y_{k} x_{k}$ for each $k \in \mathcal{K}$ where $\mathbf{y}$ and $\mathbf{x}$ are left and right positive eigenvectors of $\mathbf{G}(\mathbf{q}) \mathbf{B}^{\left(n_{0}\right)}$ associated with $\lambda_{n_{0}}(\mathbf{q})$, which, by irreducibility, are unique up to positive multiples.

\section{G. Proof of Corrolary 1}

As $\mathbf{V}$ is irreducible, Observations 1 and 2 (see also the proof) imply that $\overline{\mathbf{p}}$ corresponds to a point $\overline{\mathbf{q}} \in \partial \mathrm{F}$. Since $\mathbf{q}^{*}(\mathbf{w}) \in \partial \mathrm{F}$ for any $\mathbf{w}>0$, it follows from Theorem 3 that $\overline{\mathbf{q}}=\mathbf{q}^{*}(\mathbf{w})$ if $\mathrm{w}$ is has the form (24). Now by Observations 1 and 2 , we have $\bar{q}_{1}=\cdots=\bar{q}_{K}$. Thus, as both $g$ and its derivative $g^{\prime}$ are strictly monotonic (by (A.2) and (A.3)], we must have $\mathbf{u}(\overline{\mathbf{q}})=a 1, a>0$ and $\mathbf{G}(\overline{\mathbf{q}})=1 / \rho\left(\mathbf{B}^{\left(n_{0}\right)}\right) \mathbf{I}$. Thus, the corollary follows from Theorem $\mathbf{3}$, 


\section{REFERENCES}

[1] J. M. Aein, "Power balancing in systems employing frequency reuse," COMSAT Tech. Rev., vol. 3, no. 2, pp. 277-300, 1973.

[2] H. J. Meyerhoff, "Method for computing the optimum power balance in multibeam satellites," COMSAT Tech. Rev., vol. 4, no. 1 , pp. 139-146, 1974.

[3] H. Alavi and R. Nettleton, "Downstream power control for a spread spectrum cellular mobile radio system," in Proc. IEEE Global Communications Conference (GLOBECOM), 1982, pp. 84-88.

[4] J. Zander, "Distributed cochannel interference control in cellular radio systems," IEEE Trans. Veh. Technol., vol. 41, pp. 305-311, August 1992.

[5] — - "Performance of optimum transmitter power control in cellular radio systems," IEEE Trans. Veh. Technol., vol. 41, no. 1, pp. 57-62, February 1992.

[6] G. Foschini and Z. Miljanic, "A simple distributed autonomous power control algorithm and its convergence," IEEE Trans. Veh. Technol., vol. 42, no. 4, pp. 641-646, Nov. 1993.

[7] R. Yates, "A framework for uplink power control in cellular radio systems," IEEE J. Select. Areas Commun., vol. 13, no. 7, pp. 1341-1347, September 1995.

[8] R. Yates and C. Huang, "Integrated power control and base station assignment," IEEE Trans. Veh. Technol., vol. 44, no. 3, pp. 638-644, August 1995.

[9] N. Bambos, "Toward power-sensitive network architectures in wireless communications: Concepts, issues, and design aspects," IEEE Personal Commun. Mag., vol. 5, pp. 50-59, June 1998.

[10] T. ElBatt and A. Ephremides, "Joint scheduling and power control for wireless ad hoc networks," IEEE Trans. Wireless Commun., vol. 3, no. 1, pp. 74-85, January 2004.

[11] J. Zander and S.-L. Kim, Radio Resource Management for Wireless Networks. Artech House, Boston, London, 2001.

[12] W. Yang and G. Xu, "Optimal downlink power assignment for smart antenna systems," in Proc. IEEE International Conference on Acoustics, Speech, and Signal Processing (ICASSP), Seattle, WA, USA, May 1998.

[13] M. Schubert and H. Boche, "Qos-based resource allocation and transceiver optimization," Foundation and Trends in Communications and Information Theory, vol. 2, no. 6, 2006.

[14] H. Boche and M. Schubert, "Resource allocation in multiantenna systems - Achieving max-min fairness by optimizing a sum of inverse SIRs," IEEE Trans. Signal Processing, vol. 54, no. 6, June 2006.

[15] S. Stanczak, M. Wiczanowski, and H. Boche, Fundamentals of Resource Allocation in Wireless Networks, ser. Foundations in Signal Processing, Communications and Networking. Springer, Berlin, 2009, vol. 3, (to appear).

[16] S. Kandukuri and S. Boyd, "Optimal power control in interference-limited fading wireless channels with outage-probability specifications," IEEE Trans. Wireless Commun., vol. 1, no. 1, pp. 46-55, Jan. 2002.

[17] J. Papandriopoulos, J. Evans, and S. Dey, “Optimal power control for rayleigh-faded multiuser systems with outage constraints," IEEE Trans. Wireless Commun., vol. 4, no. 6, pp. 2705-2715, Nov. 2005.

[18] — - "Outage-based optimal power control for generalized multiuser fading channels," IEEE Trans. Commun., vol. 54, no. 4, pp. 693-703, April 2006.

[19] S. Borst and P. Whiting, "Dynamic channel-sensitive scheduling algorithms for wireless data throughput optimization," IEEE Trans. Veh. Technol., vol. 52, no. 3, pp. 569-586, May 2003.

[20] J.-W. Lee, R. Mazumdar, and N. Schroff, "Oportunistic power scheduling for dynamic multi-server wireless systems," IEEE Trans. Wireless Commun., vol. 5, no. 6, pp. 1506-1515, June 2006. 
[21] M. J. Neely, "Dynamic power allocation and routing for satellite and wireless networks with time varying channels," Ph.D. dissertation, Massachusetts Institute of Technology, LIDS, Cambridge, MA, USA, November 2003.

[22] M. Neely, C.-P. Lee, and E. Modiano, "Fairness and optimal stochastic control for heterogeneous networks," in Proc. 23rd IEEE Conference on Computer Communications (INFOCOM), Miami, FL, USA, March 13-17 2005.

[23] A. Stolyar, "Maximizing queueing network utility subject to stability," Queueing Systems, vol. 50, no. 4, pp. 401-457, Aug. 2005.

[24] D. Gerlach and A. Paulraj, "Base station transmitting antenna arrays for multipath environments," Signal Processing (Elsevier Science), vol. 54, pp. 59-73, 1996.

[25] B. He, M. Wang, and E. Li, "A new distributed power balancing algorithm for CDMA cellular systems," in Proc. IEEE International Symposium on Circuits and Systems (ISCAS), vol. 3, 1997, pp. 1768 - 1771.

[26] W. Hongyu, H. Aiging, H. Rong, and G. Weikang, "Balanced distributed power control," in IEEE International Symposium on Personal, Indoor and Mobile Radio Communications (PIMRC), vol. 2, 2000, pp. 1415 - 1419.

[27] G. Montalbano, I. Ghauri, and D. T. M. Slock, "Spatio-temporal array processing for CDMA/SDMA downlink transmission," in Proc. Asilomar Conf. on Signals, Systems and Computers, Monterey,CA,USA, 1998, pp. 1337-1341.

[28] H. Mahdavi-Doost, M. Ebrahimi, and A. K. Khandani, "Characterization of rate region in interference channels with constrained power," in Proc. IEEE International Symposium on Information Theory (ISIT), Nice, France, 2007.

[29] A. Feistel, S. Stanczak, and M. Kaliszan, "Resource allocation in multiantenna systems under general power constraints," in Proc. of International ITG Workshop on Smart Antennas 2009 (WSA), Berlin, Germany, Feb. 16-19 2009.

[30] S. Stanczak, M. Wiczanowski, and H. Boche, "Distributed utility-based power control: Objectives and algorithms," IEEE Trans. Signal Processing, vol. 55, no. 10, pp. 5058-5068, Oct. 2007.

[31] P. Hande, S. Rangan, M. Chiang, and X. Wu, "Distributed uplink power control for optimal sir assignment in cellular data networks," IEEE/ACM Trans. Networking, vol. 16, no. 6, pp. 1420-1433, Dec. 2008.

[32] R. Horn and C. Johnson, Matrix Analysis. Cambridge University Press, 1985.

[33] C. D. Meyer, Matrix Analysis and Applied Linear Algebra. SIAM, Philadelphia, 2000.

[34] S. Boyd and L. Vandenberghe, Convex Optimization. Cambridge University Press, 2004.

[35] S. Stanczak and H. Boche, "Strict log-convexity of the minimum power vector," in Proc. IEEE International Symposium on Information Theory (ISIT), Seattle, WA, USA, July 9-14 2006.

[36] D. Bertsekas and R. Gallager, Data Networks. Prentice-Hall, Englewood Cliffs, 1992.

[37] J. Mo and J. Walrand, "Fair end-to-end window-based congestion control," IEEE/ACM Trans. on Networking, vol. 8, no. 5, pp. 556-567, October 2000.

[38] C. Tan, M. Chiang, and R. Srikant, "Fast algorithms and performance bounds for sum rate maximization in wireless networks," in Proc. 28th IEEE Conference on Computer Communications (INFOCOM), Rio de Janeiro, Brazil, April, 19-25 2009.

[39] S. Stanczak, M. Kaliszan, N. Bambos, and M. Wiczanowski, "A characterization of max-min SIR-balanced power allocation with applications," 2008, available at http://arxiv.org/abs/0901.0824.

[40] M. Chiang, P. Hande, T. Lan, and C. Tan, "Power control in wireless cellular networks," Found. Trends Netw., vol. 2, no. 4, pp. 381-533, 2008.

[41] S. Friedland and S. Karlin, "Some inequalities for the spectral radius of non-negative matrices and applications," Duke Math. J., vol. 42, no. 3, pp. 459-490, 1975. 
[42] E. Deutsch and M. Neumann, "Derivatives of the Perron root at an essentially nonnegative matrix and the group inverse of an M-matrix,” J. Math. Anal. Appl., vol. I-29, no. 102, pp. 1-29, 1984. 